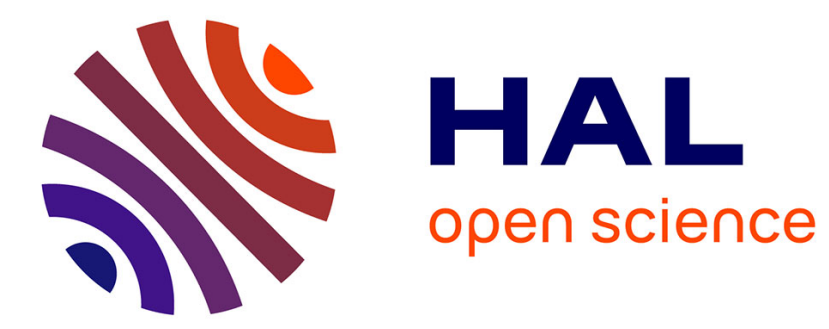

\title{
Documents, Objet et Recherche Historique
}

Maëlle Ramage

\section{To cite this version:}

Maëlle Ramage. Documents, Objet et Recherche Historique. Revue de Synthèse, 2009, 130 (4), pp.697-703. 10.1007/s11873-009-0095-2 . hal-00545752

\section{HAL Id: hal-00545752 \\ https://hal.science/hal-00545752}

Submitted on 12 Dec 2010

HAL is a multi-disciplinary open access archive for the deposit and dissemination of scientific research documents, whether they are published or not. The documents may come from teaching and research institutions in France or abroad, or from public or private research centers.
L'archive ouverte pluridisciplinaire HAL, est destinée au dépôt et à la diffusion de documents scientifiques de niveau recherche, publiés ou non, émanant des établissements d'enseignement et de recherche français ou étrangers, des laboratoires publics ou privés. 


\title{
DOCUMENTS, OBJET ET RECHERCHE HISTORIQUE À propos d'un procès de canonisation au Moyen Âge
}

\author{
Maëlle RAMAGE*
}

L e 23 mai 1325, les Augustins de Tolentino, localité de la Marche d'Ancône, obtiennent du pape une bulle autorisant enfin l'ouverture du procès de canonisation de l'un des leurs, Nicolas de Tolentino, mort le 10 septembre 1305. Dès lors, les instigateurs du procès travaillent à produire la documentation qui doit prouver la sainteté de cet homme. Les archives qui en résultent sont au centre de la recherche de Didier Lett et déterminent le cadre spatial et temporel et la composition de la société étudiée dans son livre Un procès de canonisation au Moyen Âge. Essai d'histoire sociale. Mais l'analyse de la construction d'une société, la " société du procès ", concomitante de celle d'une documentation qui seule aujourd'hui nous permet de l'appréhender, ne compose pas la totalité de l'ouvrage; au fil du récit est également exposée la réflexion qui a permis de mener à bien cette recherche. Cela tient sans doute au type de travail universitaire dont est issu l'ouvrage, une habilitation à diriger des recherches soutenue en décembre 2006, mais introduit un parti pris novateur.

Les documents sont en effet étudiés non pas en fonction de leur contenu positif, des récits de miracles et une vie de saint, mais comme le résultat d'une entreprise menée dans le but de faire canoniser Nicolas de Tolentino, dans le cadre réglementé d'une procédure inquisitoire organisée par la papauté. De ce fait, la question de la sainteté est déplacée dans le processus du procès, pour être étudiée non comme la raison de son ouverture, comme c'est souvent le cas dans l'historiographie traditionnelle, mais comme son produit: sainteté et documentation sont donc l'aboutissement du procès, la documentation est efficace au sein même de la société qui la produit.

Cette documentation consiste principalement en deux manuscrits rédigés par les copistes de la curie avignonnaise à partir des procès verbaux, non conservés, de l'enquête qui doit prouver la sainteté de Nicolas de Tolentino. Dans la première partie, intitulée Documenta et mandata, sont transcrites les principales lettres qui ont abouti à l'ouverture du procès. Viennent ensuite vingt-deux Articuli interrogatori, grilles à partir desquelles les enquêteurs orientent les questions posées aux déposants, puis

* Sur Didier LetT, Un procès de canonisation au Moyen Âge. Essai d'histoire sociale: Nicolas de Tolentino, 1325, Paris, Presses universitaires de France (Le nœud gordien), 2008, 473 p. Maëlle Ramage, née en 1982, ancienne élève de l'École supérieure d'architecture de Nantes, est actuellement doctorante et allocataire-monitrice à l'université de Paris 1, au Laboratoire de médiévistique occidentale de Paris (LAMOP, Université Paris 1, Panthéon-Sorbonne, 17 rue de la Sorbonne, 75232 Paris cedex 05 ; maelle.ramage@univ-paris1.fr). Elle y poursuit sa thèse sur la formation de la communauté de la ville de Cavaillon du XIII ${ }^{\mathrm{e}}$ au début du XV ${ }^{\mathrm{e}}$ siècle sous la direction de Claude Gauvard. Ses recherches portent principalement sur les rapports entre espace et écrit à la fin du Moyen Âge. 
les Citationes et juramenta testium dans lesquels les comparutions des témoins sont décrites. Enfin, sont consignées 371 dépositions. Comme la procédure le leur impose, les copistes de la curie pontificale ont également réalisé autour de 1330 un « résumé » du procès nommé Abbreviatio maior ou Recollectio maior dont six manuscrits sont conservés. L'analyse de la société du procès se fait par la déconstruction des documents et la restitution des étapes de leur réalisation dans un dialogue constant avec son contexte historique plus ou moins large. Il nous importera surtout ici de chercher à comprendre en quoi l'insertion au premier plan de la question documentaire, objet d'une microanalyse, apporte non seulement une relecture du dossier hagiographique, mais plus largement, des éléments de renouvellement des méthodes de l'histoire sociale.

Le livre est découpé en sept parties, regroupées selon trois séquences qui correspondent à trois niveaux d'observation du document. Les deux premières parties embrassent l'ensemble du procès, depuis les préalables à son ouverture jusqu'à la canonisation en 1346-1347 dans une optique très large, tandis que la troisième partie opère un changement d'échelle radical puisqu'elle consiste en une observation au plus près des documents. De nouveau, dans les quatre dernières parties consacrées à l'analyse de la société donnée à voir par les documents, la focale s'élargit progressivement, depuis le choix des témoins et l'écriture des dépositions jusqu'aux mécanismes sociaux à l'œuvre dans le groupe étudié.

En considérant la sainteté comme une construction du procès, Didier Lett oblige dans un premier temps à poser la question des intérêts d'une telle production, d'autant plus que mener le procès à bien est difficile et coûte très cher. La lecture des documenta et mandata montre que les Augustins sont soutenus par les autorités locales, laïques et ecclésiastiques, et par les grandes familles guelfes dans cette entreprise qui revêt des enjeux à la fois sociaux, politiques et financiers. L'auteur démontre en outre que ces enjeux, plus qu'une éventuelle popularité de l'ermite, sont à la base de l'entreprise, remettant ainsi en question l'explication traditionnelle de l'échec du procès sous le pontificat de Jean XXII, pour mettre en avant la faiblesse du culte et de la popularité de Nicolas dont la fête n'est célébrée qu'à partir de 1399, soit plus de cinquante ans après sa canonisation.

Les demandes répétées des Augustins aboutissent en 1324 avec la bulle d'ouverture du procès. Mais ni les soutiens politiques et financiers dont ils disposent, ni la faveur du pape pour leur ordre ne peuvent suffire à faire canoniser Nicolas de Tolentino, encore faut-il convaincre de son efficacité pour tous. Sur ce point, les miracles dont font état les déposants montrent comment les Augustins parviennent à fabriquer une « image performante » qui repose à la fois sur les stéréotypes de l'hagiographie (miracles attribués au Christ), les débats de la curie avignonnaise (pauvreté et érémitisme) et la situation des Marches (luttes entre guelfes et gibelins). Le renversement de la question de la sainteté permet de sortir des schémas traditionnels de l'histoire hagiographique pour remettre en perspective les enjeux propres à ce procès dans une optique d'histoire sociale. Les acteurs du procès ne sont plus alors vus comme un groupe homogène de croyants mais comme une société complexe et hiérarchisée; l'un des enjeux de la recherche consiste en une compréhension des formes de domination qui aille au-delà d'une vision réductrice opposant «dominants » et «dominés ». Ainsi, à travers les documents, ce sont 
les formes diverses de rapports sociaux - domination d'instances politico-religieuses lointaines, jeu des puissances locales, intérêts économiques, rapports de sexe - et leur interaction qu'analyse Didier Lett.

C'est pourquoi, dans la troisième partie, chaque étape de la production des documents, depuis l'obtention de la bulle jusqu'à l'achèvement de l'Abbreviatio maior, est décortiquée pour démontrer comment les parties en présence, papauté et requérants, font en sorte de retirer le maximum de bénéfice du procès. Loin de se contenter de l'étude des informations contenues dans les textes, Didier Lett met à profit les apports de la diplomatique, de la codicologie et de l'anthropologie afin de comprendre « les relations qu'entretiennent les pratiques et les discours pour éviter de les confondre ${ }^{1} »$. Les documenta et mandata relatent, entre autres préalables au procès, la constitution d'une commission d'enquête qui permet à la papauté d'afficher son alliance avec l'épiscopat guelfe, tandis que les requérants élisent deux procureurs parmi les notables et les Augustins de Tolentino. Si l'enquête sur la sainteté de Nicolas de Tolentino est une procédure inquisitoire telle qu'elle se développe à partir du XIII ${ }^{\mathrm{e}}$ siècle, lancée par une dénonciation initiale, elle vise à établir la vérité; la rédaction dès le 23 mai par les requérants des articuli interrogatori montre l'importance des questions posées pour maîtriser la construction de l'image du saint pendant l'enquête.

Les citationes et iuramenta testium renseignent sur le déroulement des interrogatoires du 23 juillet au 28 septembre 1325. En comparant les différentes étapes de l'enquête, Didier Lett relève une forte déperdition entre les personnes citées à comparaître (668), le nombre de témoins effectivement interrogés (434) et les dépositions enregistrées (332). Cet écrémage aboutit à un rééquilibrage masculinité/féminité et à une plus forte proportion de notables. Étant donnée la rigueur de l'enquête, il est clair que de nombreux témoignages n'ont pas été perdus mais bien écartés car ils n'étaient pas conformes au discours à produire. L'analyse graphique des manuscrits et de l'ordonnancement des témoins prend toute sa place dans la démonstration en ce qu'elle permet de dépasser l'idée d'une communauté de chrétiens alliés dans une entreprise visant à faire reconnaître une sainteté en laquelle ils ont foi au préalable, pour commencer à esquisser le portrait de la société constituée autour du procès. La mise en page des manuscrits révèle la hiérarchie sociale des lieux visités par les enquêteurs. Par exemple, l'inscription de la liste des notables et des Augustins de Tolentino appelés à témoigner fait l'objet d'un soin particulier et est séparée de celle des autres déposants; le scribe est passé à la page suivante pour commencer à inscrire ces derniers alors que plusieurs lignes sont vierges à la suite de la liste des notables. Après les notables viennent les hommes porteurs d'un titre, puis ceux qui n'en ont pas, et enfin les femmes. La comparaison des structures graphiques des manuscrits et de l'Abbreviatio indique que la mise en avant des miracles et du saint par rapport aux miraculés et aux témoins dans le premier document a influencé le travail des rédacteurs de l'Abbreviatio. Ils ont ordonné différemment les témoignages et la logique sociale du manuscrit du procèsverbal cède la place à une stratégie de définition de la sainteté de Nicolas de Tolentino par le rassemblement des témoignages se rapportant à un même événement. À la suite des témoignages sur la vie et la mort du saint sont enregistrés ses miracles in vita puis 
post mortem. Cette synthèse rubriquée sert la construction de la preuve par la création d'effets de concordance liés à la répétition d'un même miracle.

L'analyse de la structure des documents, qui sont autant de réécritures successives du procès, montre que pour construire une vérité et des effets de vraisemblance convaincants, un témoignage n'en vaut pas un autre et que l'effet de vérité tient également à l'accumulation de discours concordants. Le travail de Didier Lett dans cette troisième partie démontre que la prise en compte du document comme résultat permet d'y rechercher bien plus qu'une somme d'informations factuelles; il offre la possibilité de remonter le fil des processus sociaux à l'œuvre dans le procès de canonisation de Nicolas de Tolentino. Concernant la société du procès, les documents donnent accès à « des personnes, des paroles, des espaces et à des inégalités sociales », objets des quatre parties suivantes ${ }^{2}$.

La société donnée à voir dans le procès résulte elle aussi de la volonté de faire canoniser Nicolas de Tolentino. Les témoins sont en effet choisis de manière à prouver que le saint est utile à tous, ce sont donc des hommes et des femmes, des notables et des humbles, des clercs et des laïcs qui sont rassemblés pour l'enquête, mais le choix des témoignages se porte favorablement sur la parole des notables et des clercs au détriment du petit peuple, des marchands et des artisans. L'analyse du statut des déposants introduit la question de la valeur testimoniale des témoins, sans laquelle il serait hasardeux d'interpréter les discours prêtés aux déposants, discours dont la très grande cohérence s'explique par les conditions de leur énonciation et de leur enregistrement. D'une part, la difficulté à se souvenir d'événements parfois anciens est palliée par la mémoire collective: les déposants se souviennent de ce qui doit en général arriver quand on invoque un saint, quand on ne leur demande pas simplement de confirmer un miracle. D'autre part, le passage de l'oral à l'écrit, de la langue vulgaire au latin, sert les requérants puisque le notaire a la possibilité de standardiser l'écriture des témoignages, créant ainsi de la cohérence. Cette réflexion sur l'écriture des témoignages remet en question l'idée de ceux-ci comme accès direct à la parole prononcée.

Les stratégies mises en place lors de l'enquête ne contribuent pas seulement à construire une vérité acceptable, elles amènent également les personnes interrogées à penser selon des schémas explicatifs et des cadres de références communs. L'espace et le temps sont ici analysés comme des constructions sociales et on constate par exemple la création d'un calendrier propre à la société du procès, avec pour point de repère principal la mort de Nicolas de Tolentino. L'efficacité sociale du procès est analysée dans les deux dernières parties qui traitent respectivement de l'espace du procès et de la domination sociale qui s'y exprime et s'y renforce. L'espace, construit par l'itinéraire de l'enquête et la parole des déposants, est étudié comme un élément propre à accueillir la renommée de Nicolas de Tolentino pour les besoins de la procédure. Il décrit des cercles concentriques autour de Tolentino depuis des villes peu citées et éloignées, où la fama de Nicolas est faible, jusqu'à Tolentino, lieu de sacralité maximum. L'expression de cette sacralité atténue les différences sociales et tend vers la société désincarnée de l'Abbreviatio, soit un groupe de chrétiens rassemblés autour des vertus d'un saint.

2. LETT, 2008, p. 183. 
Mais l'étude du temps de parole des déposants et des sujets sur lesquels ils sont ou non interrogés met en exergue une hiérarchie qui place au premier rang les clercs et les notables de Tolentino. Cette prééminence s'exprime dans les documents par des dépositions plus longues et mises en page avec soin et surtout par la possibilité de parler de la fama sanctitatis de Nicolas. Son expression, essentielle pour contrôler la renommée de ce dernier, est l'apanage des Augustins et des notables qui, de plus, sont les premiers à parler. Les miracles sont une occasion de faire parler plus de monde, mais là encore se retrouve une distinction sociale entre ceux qui racontent un ou plusieurs miracles et ceux qui ne font que les confirmer. Exprimer la fama, accumuler les récits de miracles et parler le premier, en jouant sur ces trois critères les notables et les Augustins ont su faire du procès le lieu de défense et de renforcement de leurs intérêts particuliers.

Un procès de canonisation au Moyen Âge paraît alors que l'historiographie médiévale française est marquée par un intérêt accru pour la question documentaire, déjà manifesté par la microhistoire, et par la continuité des interrogations sur l'écriture de l'histoire et le statut de la discipline depuis plus de vingt ans. Mais là où les précédentes recherches mobilisant une vraie réflexion sur les « sources » de l'historien avaient principalement pour objet les pratiques d'écriture et de conservation des documents, Didier Lett applique cette critique documentaire renouvelée dans une recherche dont l'objet est la structure sociale d'un groupe, appréhendée dans une perspective plus large et qui recoupe plusieurs courants historiographiques ${ }^{3}$. Aux interrogations sur les pratiques d'écriture s'ajoute ainsi une réflexion sur l'enquête et les moyens mis en œuvre et l'instrumentalisation de la procédure ${ }^{4}$. L'observation des catégories sociales met à profit les réflexions sur le genre qui tend à « rendre aux femmes la part qui leur revient dans cette société ${ }^{5}$ », mais sans omettre d'articuler cette catégorie aux autres modes d'identification que sont par exemple l'âge ou le niveau social. Le cadre géographique et temporel n'est pas défini préalablement à la constitution d'un corpus que l'on voudrait adéquat, il est au contraire révélé par l'analyse des documents. L'histoire commence avec les premières lettres de demande d'ouverture du procès recopiées dans le manuscrit, la société étudiée se compose des personnes qui y sont nommées, instigateurs du procès, enquêteurs ou encore déposants et le cadre géographique est celui de l'enquête et de la renommée de Nicolas de Tolentino. La société étudiée est donc celle qui se crée autour du procès, telle qu'elle est représentée dans les documents. Les hommes et les femmes présents dans le manuscrit sont aussi des déposants dont le rôle et les paroles rapportées ne peuvent être compris en dehors des enjeux de l'entreprise et de la structure sociale du groupe auquel ils participent. À cette optique interactionniste s'ajoute la variation des échelles d'analyse, de la société de Tolentino au contexte politique des Marches, et jusqu'aux débats théologiques de la curie avignonnaise. Avec le passage au premier plan des individus et de leurs paroles, et le contre-pied par rapport aux approches purement structurales, Didier Lett remet en jeu les éléments de la microhistoire

3. Concernant les recherches sur les pratiques d'écriture, voir par exemple la thèse de CHASTANG, 2001 ; pour un bilan historiographique sur ces travaux, voir CHASTANG, 2008.

4. Parmi les études récentes sur l'enquête et la place des témoins, voir par exemple GaUvard, dir., 2008; GARNOT, dir., 2003.

5. Sur la distinction des catégories sociales et la démarche de genre, voir LETT, 2008, p. 11. 
dont il se réclame mais en opérant un déplacement important ${ }^{6}$. Le temps, l'espace et la société considérés mais aussi la variation des échelles de l'analyse, la méthodologie mise en place et l'organisation de l'ouvrage: tous ces éléments passent par la médiation du document qui, ouvrant sur un monde social selon un point de vue spécifique, est un objet à part entière de l'étude, là où la microhistoire prend pour objet d'étude intensive un groupe social ou un individu à un moment précis - cette réflexion sur les documents et la lecture indivise du texte et du contexte de sa production permettant de dépasser l'écueil dangereux d'une institution du contexte en cadre explicatif de la documentation ${ }^{7}$.

Comme on le voit, la dimension expérimentale de la démarche, autre héritage de la microhistoire, est très importante. Loin d'être camouflée par un discours lissé, elle prend d'ailleurs toute sa place dans l'écriture de l'ouvrage, puisqu'au récit historique vient s'ajouter le récit de sa fabrication. Outre les références précises aux documents utilisés, à chaque étape l'auteur expose les raisonnements qui lui ont permis d'avancer sa recherche, donnant de cette façon la possibilité de comprendre non seulement les résultats de l'étude, ce qui est courant, mais aussi le cheminement intellectuel et les choix qui la fondent, ce qui est beaucoup plus rare. Ce faisant l'auteur donne la possibilité d'une lecture véritablement critique de son travail, il livre les éléments d'une évaluation de la méthodologie et du discours de connaissance produit.

Afin de saisir les processus d'un monde social toujours en mouvement, l'auteur a pris le parti d'une approche constructionniste des documents, échappant à la naturalisation des archives comme sources et à l'enfermement dans une perspective érudite ${ }^{8}$. Ainsi la restitution d'une réalité passée à partir d'un corpus documentaire cède la place à la compréhension des processus sociaux au moyen de l'observation minutieuse d'un document, point de vue spécifique en un moment donné. Surtout, Didier Lett a réinvesti dans sa recherche ce qui a renouvelé de manière critique l'approche de leur discipline par les historiens ces vingt dernières années, tant sur les outils et le statut des documents que sur l'écriture de l'histoire, mais dans un projet qui vise à se réinscrire dans la tradition d'histoire sociale et non plus à la faire éclater. Les focales varient, les documents sont construits, l'accent est mis sur les différenciations interindividuelles, mais l'ambition d'éclairer une société prise comme une chose, dans son ensemble, ses logiques, son fonctionnement, n'est jamais abandonnée.

\section{LISTE DES RÉFÉRENCES}

BENSA (Alban), 1996, «De la microhistoire vers une anthropologie critique », dans ReVEL (Jacques), dir., Jeux d'échelles. La micro-analyse à l'expérience, Paris, Gallimard/Le Seuil (Hautes Études), p. 39-48.

Chastang (Pierre), 2001, Lire, écrire, transcrire. Le travail des rédacteurs de cartulaires en Bas-Languedoc (XI ${ }^{e}$-XIII ${ }^{e}$ siècles), Paris, CTHS.

6. LETT, 2008, p. 9-10.

7. Sur le contexte, voir BENSA, 1996.

8. Sur l'approche constructionniste des documents, voir Morsel, 2004, p. 278; PonCET et ANHEIM, dir., 2004. 
Chastang (P.), 2008, «L'archéologie du texte médiéval. Autour de travaux récents sur l'écrit au Moyen Âge », Annales. Histoire, sciences sociales, $\mathrm{n}^{\circ} 2$, p. 245-270.

Garnot (Benoît), dir., 2003, Les Témoins devant la justice. Une histoire des statuts et des comportements, Rennes, Presses universitaires de Rennes.

Gauvard (Claude), dir., 2008, L'Enquête au Moyen Âge, Rome, École Française de Rome.

LetT (Didier), 2008, Un procès de canonisation au Moyen Âge. Essai d'histoire sociale: Nicolas de Tolentino, 1325, Paris, Presses universitaires de France.

Morsel (Joseph), 2004, «Les sources sont-elles "le pain de l'historien”? », Hypothèses 2003, Travaux de l'École doctorale d'histoire de Paris 1, Paris, Publications de la Sorbonne, p. 271-286.

Poncet (Olivier) et Anheim (Étienne), dir., 2004, Fabrique des archives, fabrique de l'histoire, $\mathrm{n}^{\circ}$ spéc. de la Revue de synthèse, t. 125. 